\title{
Development of High-Resolution TEM for Imaging Native, Radiation-Sensitive Biological Macromolecules
}

\author{
Robert M. Glaeser ${ }^{1}$ \\ 1. Molecular Biophysics and Integrated Bioimaging Division, Lawrence Berkeley National Laboratory, \\ University of California, Berkeley, 94720 USA
}

Following the commercial introduction of "direct detection" cameras about 5 years ago, single-particle electron microscopy has begun to produce atomic-resolution structures for a large number of biological macromolecules; see, for example, Figure1 and [1,2]. This important advance greatly broadens the scope of what can be learned within the fields of biochemistry, molecular biology, and cell biology. This presentation reviews some of the main developments that led up to the current capability. In addition, it explores where the current achievements stand relative to reaching the limits of what is physically possible, and what prospect there is for further narrowing the gap.

The first requirement for high-resolution electron microscopy of biological macromolecules is to maintain the native, hydrated structure during imaging. At first glance, that might seem to be something that is not compatible with putting specimens into the vacuum of an electron microscope, but surprisingly many solutions can give at least partial success. At present, the preferred approach is to use thin, unstained frozen specimens that are held at a temperature low enough to prevent loss of water by sublimation $[3,4]$.

The second requirement is to record images with electron exposures that are sufficiently low that radiation damage does not destroy the sought-after, high-resolution features. Although such images are too noisy to see high-resolution details, Frank recognized that they nevertheless can be aligned by crosscorrelation, if the particles of interest are large enough [5]. This means that image information can be merged to produce data with a much better signal-to-noise ratio (SNR). The ultimate limitations of that approach have been estimated to be that particles should be larger than $40 \mathrm{kDa}$, and that the data set should include images of more than $~ 12,000$ particles [6]. The current state-of-the-art, impressive though it is, still falls well-short of that estimate. Additional improvements in the technology of cryoEM are thus very welcome and, indeed, expected. Anticipated improvements include reliable phase plates, which have just begun to appear, and cameras whose spectral Detective Quantum Efficiency is at least $2 \mathrm{X}$-improved at high resolution.

Another area where improvement may be possible is the recovery of high-resolution signal during the earliest portion of the electron exposure, before radiation damage has become significant but during which the specimen experiences the greatest amount of beam-induced movement. It remains to be seen whether simple techniques can be devised to accomplish this, or whether more challenging, even exotic technologies have to be used, such as recording frames of movies with ultrafast exposures that might "outrun" the motion that occurs during the frame, or using multipass imaging, described in Figure 2 (https://arxiv.org/abs/1612.04931), to improve the SNR to a point that approaches the Heisenberg limit. 


\section{References:}

[1] E. Nogales, Nat Meth 13 (2016) 24.

[2] A. Merk et al, Cell 165 (2016) 1698.

[3] K. A. Taylor and R. M. Glaeser, Science 186 (1974) 1036.

[4] M. Adrian et al, Nature 308 (1984) 32.

[5] O. Saxton and J. Frank, Ultramicroscopy 2 (1977) 219.

[6] R. Henderson, Quart Rev Biophys 28 (1995) 171.

[7] C Lawson et al, Nucleic Acids Res 44 (2016) D396.

[8] The author acknowledges funding from NIH grant P01 GM051487.
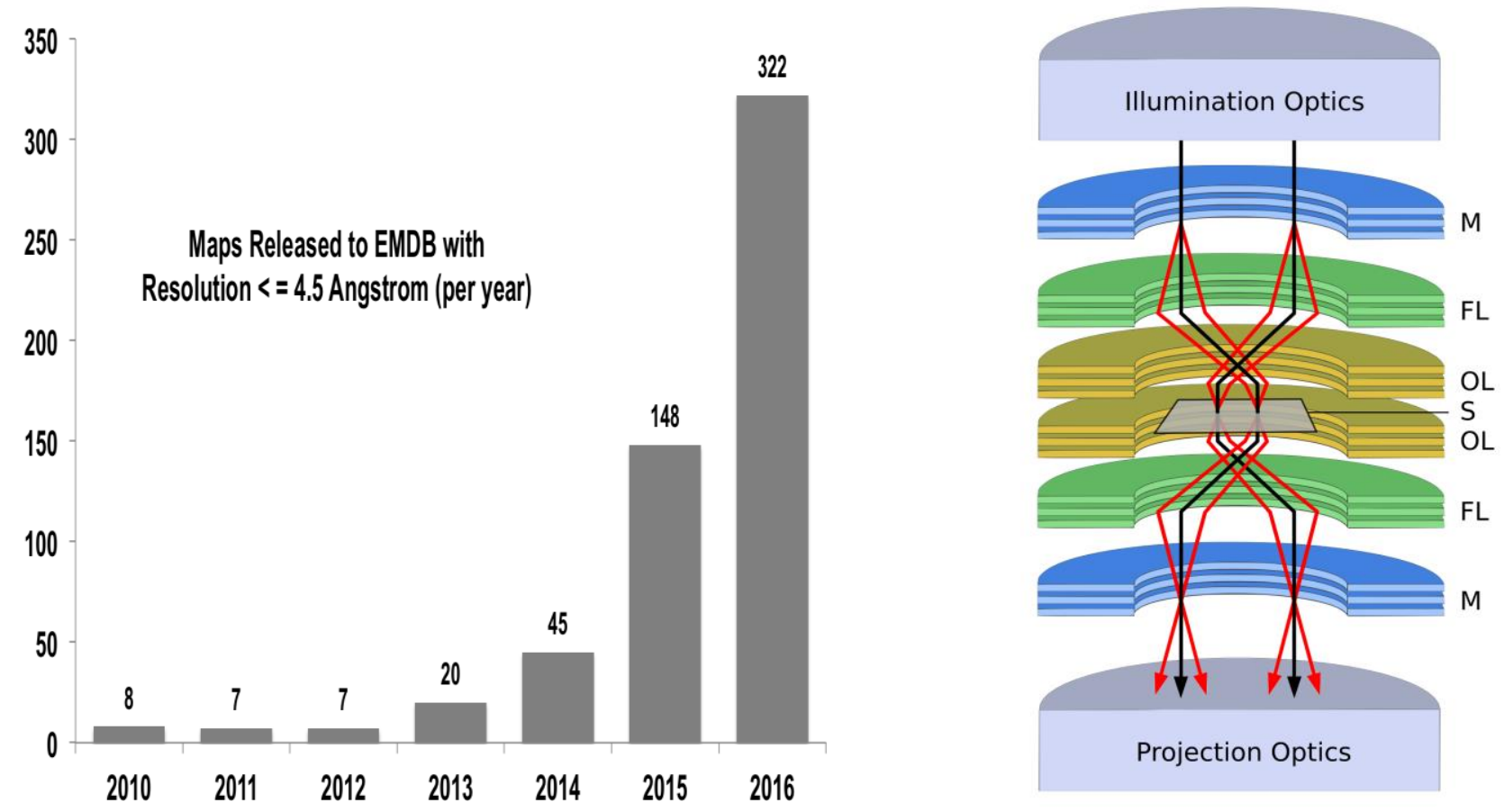

Figure 1. Recent growth in the number of high-resolution, cryo-EM structures of biological macromolecules that have been deposited in the EM Data Bank [7]. Figure provided courtesy of Dr. Cathy Lawson.

Figure 2. Cartoon representation of a scheme proposed to pass electrons multiple times through the specimen plane, indicated as " $\mathrm{S}$ ". "M" designates mirrors that can be turned on and off, "FL" field lenses, and "OL" objective lens elements. Figure taken from https://arxiv.org/abs/1612.04931. 